\title{
Trattamento immunosoppressivo della glomerulonefrite membranoproliferativa
}

\author{
R. Faedda ${ }^{1}$, A. Satta ${ }^{1}$, L. Bosincu ${ }^{2}$, M. Pirisi ${ }^{3}$, E. Bartoli ${ }^{3}$ \\ ${ }^{1}$ Istituto di Patologia Medica dell'Università di Sassari \\ ${ }^{2}$ Istituto di Anatomia Patologica dell'Università di Sassari \\ ${ }^{3}$ Cattedra di Medicina Interna dell'Università di Udine
}

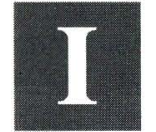

1 trattamento delle glomerulonefriti $(\mathrm{GN})$ risulta ancora controverso. I corticosteroidi divennero di uso comune dopo che fu stabilmente confermata la regressione della $\mathrm{GN}$ a lesioni minime, che poteva essere immunomediata (1), con adeguate dosi di prednisone (PDN) (2). Gli steroidi furono pertanto sperimentati in altre $\mathrm{GN}$ immunomediate. Dopo segnalazioni inizialmente favorevoli, vennero sperimentati trattamenti immunosoppressivi più complessi ed estesi. Questi includevano agenti alchilanti, anti-infiammatori ed antiaggreganti piastrinici (3).

La risposta al trattamento è sicura e non controversa (4-6) in alcune GN, mentre fornisce risultati contrastanti nella GN Membranosa (GNM). Un'ampia revisione della letteratura evidenzia schemi terapeutici assai differenti; alcuni autori hanno usato il solo PDN (7), altri solamente agenti alchilanti (8), altri basse dosi di PDN (9) o per periodi brevi di trattamento $(10,11)$, altri ancora dosi elevate (12) o trattamenti prolungati (9).

Pochi lavori combinavano PDN ad agenti alchilanti. Per questa ragione abbiamo studiato l'efficacia di un regime terapeutico immunosoppressivo basato sull'utilizzo di alte dosi di PDN associate ad alte dosi di ciclofosfamide
(CPM) per un periodo prolungato di tempo in 30 pazienti con GNM.

\section{Materiali e metodi}

Lo studio fu iniziato nel 1975 ed il reclutamento di pazienti interrotto nel 1989 , mentre il periodo di follow-up è tuttora in corso. Questo studio faceva parte di una più ampia sperimentazione del trattamento immunosoppressivo delle glomerulonefriti, sistematicamente iniziata in tutti i pazienti con Sindrome Nefrosica (NS) e successivamente estesa ai pazienti con GN Membrano Proliferativa (GNMP) e/o con entità cliniche tipo LES, porpora di Schönlein-Henoch ed altre.

A tutti i pazienti con GNM veniva richiesta l'autorizzazione a partecipare allo studio dopo essere stati informati su possibili rischi e sui non certi benefici della terapia immunosoppressiva e sul decorso spontaneo della malattia renale non trattata. Non abbiamo sottoposto a biopsia renale alcun paziente con normale funzione renale, a meno che non presentasse SN o proteinuria stabile in un range nefrosico. Di conseguenza lo studio era limitato a questi pazienti.

Noi abbiamo studiato 38 pazienti con
GNM idiopatica: 8 (5M e $3 \mathrm{~F}$ di età media di 42 anni - range 23-64) rifiutarono il trattamento per cui furono seguiti nel tempo. Essi perciò non costituiscono un vero e proprio gruppo di controllo pienamente confrontabile con la popolazione sperimentale ai fini statistici.

Trenta pazienti (16M e $14 \mathrm{~F})$ di età media di 41 anni (range 17-68) furono trattati col nostro protocollo previo consenso informato. I campioni di tessuto ottenuti con la biopsia renale furono sottoposti ad esame ottico, utilizzando sezioni ultrasottili, ad Immunofluorescenza e a Microscopia Elettronica. Il numero medio dei glomeruli ottenuti fu di 23 (range 970). La diagnosi istologica fu posta sulla base di criteri standard (12). Ciascun paziente aveva un'anamnesi particolareggiata, con esame obiettivo e relativa valutazione per apparato. Venivano effettuati su ciascun paziente analisi routinarie del sangue e delle urine, esame della funzione renale (inclusa la clearance della creatinina, capacità massima di concentrazione urinaria e test addizionali se necessari), dosaggio del complemento ed autoanticorpi per identificare la natura della GN. I pazienti venivano rivalutati ambulatorialmente ogni due settimane per i primi tre mesi dopo l'inizio della terapia, quindi mensilmente per gli altri tre mesi, e due volte l'anno successiva- 
mente. Visite più frequenti venivano effettuate solo se necessario.

Lo schema del trattamento consisteva in quattro fasi, qui sotto riportate:

1) INDUZIONE - Metilprednisolone (mPDN) da $250 \mathrm{mg}$ a $750 \mathrm{mg}$ e.v. per 36 gg, seguito da PDN 100 o 200 mg per os al dì per 3-7 gg. CPM da 100 a 200 mg per os q.d.

2) MANTENIMENTO - PDN da 100 a $200 \mathrm{mg}$ per os a giorni alterni, associato all'agente alchilante allo stesso dosaggio, come nelle fasi di induzione e ridotto solamente se la conta dei GB cadeva al di sotto di 1.500/ul. Questa fase durava circa 45-75 giorni.

3) RIDUZIONE - Il PDN veniva ridotto di $25 \mathrm{mg}$ al mese fino ad un dosaggio di 25-50 mg per os a giorni alterni. Se dopo aver ridotto la dose, la proteinuria aumentava, il PDN veniva riportato alle dosi di 100-150 mg per os a giorni alterni per 7-14 giorni e quindi ridotto più lentamente (in genere del $50 \%$ della dose prevista dallo schema).

4) FASE DI SOSPENSIONE - Se il paziente era in fase di remissione clinica, la riduzione veniva prolungata fino a raggiungere $15-25 \mathrm{mg}$ per os a giorni alterni. A questo punto la CPM veniva sospesa e il PDN ridotto fino a zero secondo lo schema di sospensione steroidea. Il PDN veniva somministrato a colazione e a pranzo. Se non si aveva miglioramento clinico o se si presentava una ripresa di malattia durante tale trattamento, si intraprendeva un nuovo ciclo di induzione con dosi massimali, seguite da una fase più corta di mantenimento.

Il trattamento veniva personalizzato al singolo individuo, aggiustando la dose entro il range sopra riportato (la dose media veniva riferita a $1.5 \mathrm{~m}^{2}$ di superficie corporea).

Pazienti con GNM pura, con normale FG e proteinuria inferiore a $8 \mathrm{~g}$ non ricevevano boli di mPDN. Questi venivano fatti in pazienti con perdite proteiche superiori agli $8 \mathrm{~g}$ e/o quando la biopsia renale presentava lesioni infiammatorie in aggiunta ai depositi epimembranosi.

Le dosi di mantenimento erano nel range superiore quando il paziente aveva una SN conclamata (edema, proteinuria $>3$ g/die, ipoalbuminemia e iperlipidemia), quando c'era anasarca, quando la funzione renale si deteriorava e quando la biopsia renale evidenziava componenti infiammatorie ed essudative.

Sebbene qualche biopsia renale eviden- ziasse depositi epimembranosi associati ad aspetti essudativi, nessuno dei nostri pazienti aveva un LES. Pazienti con LES trattati con un ciclo similare, sono stati pubblicati separatamente.

Test di laboratorio - Tutti i test venivano eseguiti in un laboratorio centralizzato usando procedure standard, eccetto per l'analisi delle urine e del sedimento urinario che veniva personalmente preparato e valutato da noi. I test immunologici venivano eseguiti in un laboratorio di ricer- ca. La conta spermatica veniva fatta su tutti i pazienti maschi. Nelle femmine venivano dosate le gonadotropine e gli estrogeni ed esaminate ecograficamente le ovaie per fibrosi. Le biopsie renali furono sempre attentamente valutate da noi insieme all'anatomo-patologo.

Abbiamo stilato dei criteri diagnostici anatomo-patologici, e su tali parametri (riportati nella Tab. I) rivalutato il preparato istologico, senza informazioni sul caso clinico, prima della pubblicazione

TAB. I - CARATTERISTICHE ISTOLOGICHE DELLE BIOPSIE RENALI IN PAZIENTI CON GNM

\section{Membrana basale}

Tipo di lesione
A) ISPESSIMENTO
B) Sfilacciamento
C) Festonatura
D) DEPOSITI

\section{E) IMMUNOFLUORESCENZA}

\author{
Massivo e diffuso \\ Variabile e focale
}

Subepiteliali Subendoteliali Intramembranosi IgG
IgM
IgA
C $_{3}$ solamente
$\mathrm{C}_{3}$
$\mathrm{C}_{4}$
Fibrinogeno

\section{Proliferazione mesangiale}

Modesta
VARIABILE
DifFUSA

\section{Leucociti marginati}

Lungo le anse capillari (in media 1 ogni 4 glomeruli)

\section{Semilune}

5. Adesioni flocculo-capsulari

6. Infiltrati interstiziali

7. Glomeruli jalini (in media 2 per biopsia - range $0-12$ )
$\%$ dei pazienti

80
20
23
2

100
-
6
77
13
16
10
30
6
3

$\%$ dei pazienti 30 13 6

$\%$ dei pazienti 17

$\%$ dei pazienti 6 13 20 30
La tabella riporta le percentuali dei pazienti con GNM che avevano lesioni istologiche nelle loro biopsie renali. 3, 4 e 5 erano considerate lesioni essudative. Le lesioni da 4 a 7 nello stesso campione erano considerate indicative di lesione cronica. Gli infiltrati interstiziali, accompagnati da atrofia tubulare e fibrosi, erano presenti intorno a nefroni obsoleti nelle aree di glomeruli jalini. 
del lavoro. Le definizioni cliniche venivano stabilite prima della diagnosi e del trattamento ed erano usate nel testo come indicato sotto:

- REMISSIONE - Esame delle urine negativo, funzione renale normale, escrezione delle proteine urinarie sotto i 300 $\mathrm{mg} / \mathrm{die}$, esame fisico del paziente negativo.

Se il paziente aveva IRC prima del trattamento (P Creat $>150 \mu \mathrm{mol} / \mathrm{L}$ - U Osm $\max <800 \mathrm{mOsm} / \mathrm{kg} \mathrm{H}_{2} \mathrm{O}$, glomeruli ialini $>20 \%$ ), veniva considerato in remissione anche se la funzione renale ed il sedimento urinario non presentavano un rientro alla norma, ma solamente un miglioramento (vedi sotto), purché l'albumina plasmatica aumentasse significativamente e la proteinuria cadesse almeno al di sotto del $50 \%$.

- MIGLIORAMENTO - (Parziale Remisione). P Creat immodificata o ridotta almeno di $30 \mu \mathrm{mol} / \mathrm{L}$ se prima era sopra $\mathrm{i}$ $100 \mu \mathrm{mol} / \mathrm{L}$, proteine urinarie ridotte di più del $50 \%$ con un concomitante aumento sierico dell'albumina, con miglioramento o scomparsa dell'edema.

- NESSUN MIGLIORAMENTO - Proteinuria e creatinina immodificate indipendentemente dal miglioramento dei rimanenti parametri.

- PROGRESSIONE - Aumento progressivo della creatinina o sua stabilizzazione oltre $30 \mu \mathrm{mol} / \mathrm{L}$ al disopra della concentrazione del pre-trattamento.
- INSUFFICIENZA RENALE - Aumento della creatininemia al di sopra di 500 $\mu \mathrm{mol} / \mathrm{L}$ rispetto ai valori del pre-trattamento.

- RECIDIVA - Aumento progressivo della proteinuria con cilindruria. L'aumento isolato della creatinina dopo la remissione non veniva considerato ricaduta, ma solo progressione. Questo stretto criterio per definire la ripresa della malattia era stato introdotto per evitare di etichettare come miglioramento o remissione un occasionale o breve periodo di riduzione nella proteinuria al di sotto dei 300 $\mathrm{mg} / \mathrm{die}$.

Noi pertanto abbiamo voluto essere sicuri prospetticamente, che tutte le remissioni fossero reali. In caso di progressione verso l'Insufficienza Renale terminale, sarebbe stato importante poter discriminare i pazienti con apparente remissione da quelli con una chiara ripresa di malattia (proteinuria e sedimento anomalo) onde evitare di valutare come ripresa di malattia la progressione verso l'IR.

Le SN furono definite da proteinuria persistente superiore a $3 \mathrm{~g} /$ die o tali da causare edema e/o ipoalbuminemia. Questa definizione è leggermente differente, ma è più circostanziata di quella usualmente accettata. L'abbiamo ritenuta necessaria per evitare di definire come remissione la riduzione della proteinuria secondaria ad ipoalbuminemia.

Infatti una remissione richiede la com- pleta scomparsa della proteinuria o una sua caduta del $50 \%$ o più, in associazione con l'aumento significativo delle proteine plasmatiche.

Prima di pubblicare il nostro lavoro, tutte le biopsie renali sono state sottoposte a rivalutazione dagli Autori, e le diagnosi e la descrizione analitica delle lesioni riscritte. Solamente i casi in cui nefologi e gli anatomopatologi si trovavano d'accordo sono stati inclusi nel lavoro.

I dati sono stati analizzati statisticamente; sono state calcolate medie e deviazione standard, e la loro significatività è stata testata con il "t" di Student.

\section{Risultati}

Noi abbiamo studiato 30 pazienti le cui caratteristiche istologiche sono state riportate in Tabella I; 47\% appartenevano al II stadio, $48 \%$ al III stadio e il $5 \%$ al IV.

Come evidenziato dalla Tabella II, abbiamo ottenuto 25 remissioni complete (83\%), 3 parziali e due progressioni. Nove pazienti che avevano recidivato con aumento della proteinuria fino ai valori iniziali, furono sottoposti ad un secondo ciclo di terapia con piena remissione.

Un terzo ciclo di terapia fu intrapreso in un paziente già responsivo ai precedenti trattamenti, in occasione della seconda

TAB. II - EFFETTI DEL TRATTAMENTO IMMUNOSOPPRESSIVO IN 30 PAZIENTI CON GNM

\begin{tabular}{lccccc}
\hline Numero dei pazienti & Trattati & $\begin{array}{c}\text { Completa } \\
\text { remissione }\end{array}$ & Miglioramenti & $\begin{array}{c}\text { Progressione } \\
\begin{array}{c}\text { Ripresa di } \\
\text { malattia }\end{array}\end{array} \begin{array}{c}\text { Remissione dopo } \\
\text { ripetuti cicli }\end{array}$ \\
\hline Totale & 30 & 25 & 3 & -2 & 10 \\
P Creat $>200 \mu \mathrm{mol} / \mathrm{L}$ & 4 & 4 & 2 & - & 2 \\
Proteinuria $>6 \mathrm{~g} / \mathrm{die}$ & 9 & 6 & - & 1 & 5 \\
Proteinuria $<3.5 \mathrm{~g} / \mathrm{die}$ & 10 & 9 & & 2 & 2 \\
\hline
\end{tabular}

Media mesi di follow-up

Durata di remissione o progressione

Intervallo dalla

Durata della remissione remissione

$\begin{array}{lc}\text { Totale } & 109 \\ \text { P Creat }>200 \mu \mathrm{mol} / \mathrm{L} & 144 \\ \text { Proteinuria }>6 \mathrm{~g} / \mathrm{die} & 102 \\ \text { Proteinuria }<3.5 \mathrm{~g} / \mathrm{die} & 88\end{array}$

I risultati del trattamento sono riportati in numeri assoluti con, da sinistra verso destra, il tempo di osservazione media, la durata della remissione, l' intervallo fra remissione e recidive, che furono 10 in 9 soggetti. 
recidiva. Tale ciclo, fu intrapreso tardivamente (dopo 14 mesi) a causa di epatotossicità, quando la $\mathrm{P}$ creatinina aumentò da $106 \mu \mathrm{mol} / \mathrm{L}$ a $159 \mu \mathrm{mol} / \mathrm{L}$. Al termine la $\mathrm{P}$ creatinina si ridusse a valori di $123 \mu \mathrm{mol} / \mathrm{L}$ e la proteinuria passò da 5 g/die a $1.5 \mathrm{~g} /$ die. Dopo la remissione la creatininemia aumentò progressivamente, attestandosi su valori di $177 \mu \mathrm{mol} / \mathrm{L}$, mentre la proteinuria rimase stabile.

Non vi furono casi di Insufficienza Renale terminale né decessi per cause renali. Nel gruppo di controllo, un paziente positivo per HBsAg, con Cirrosi Epatica, morì per sanguinamento da varici esofagee.

Un paziente trattato morì per carcinoma polmonare dopo un anno di remissione. Quattro dei pazienti trattati presentavano I.Renale pre-terapia (P creat $>200$ $\mu \mathrm{mol} / \mathrm{L})$; di questi, tre normalizzarono il valore della creatinina con scomparsa della proteinuria, il quarto raggiunse la remissione con leggero miglioramento dei valori di creatinina ed una riduzione della proteinuria da $7 \mathrm{~g} / \mathrm{die}$ a $1.4 \mathrm{~g} / \mathrm{die}$.

In nove pazienti la proteinuria si ridusse da $7 \mathrm{~g} /$ die a $1.4 \mathrm{~g} /$ die, uno presentò progressione verso I. Renale, sebbene la sua creatinina sia ancora al di sotto di 200 $\mu \mathrm{mol} / \mathrm{L}$ dopo 11 anni; due migliorarono mantenendo una $\mathrm{P}$ creatinina normale ed una proteinuria di $0.5 \mathrm{~g} /$ die e $1.5 \mathrm{~g} / \mathrm{die}$. I rimanenti sei pazienti sono tuttora in completa remissione.

Dieci pazienti avevano una proteinuria inferiore ai $3.5 \mathrm{~g} / \mathrm{die}$ : uno progredì, uno migliorò, ed otto andarono in remissione. Pertanto né il grado di proteinuria, né la funzione renale ebbero predittività sul tipo di risposta al trattamento e sul successivo decorso. La durata media di remissione fu di 81 mesi. L'intervallo medio fra la remissione e la recidiva fu di 52 mesi. La dose media cumulativa per ciclo fu di $7.8 \mathrm{~g}$ per il PDN e di 27.5 g per la CPM. La durata media del ciclo fu di 9 mesi (range 5-12) dovuta alla necessità di un breve periodo di re-induzione (descritto nella sezione del metodo) in nove pazienti.

La Tabella III mostra i valori di creatinina, proteinuria, colesterolemia e trigliceridemia prima del trattamento, al termine dei 6 mesi del ciclo ed alla fine del follow-up.

Mentre la funzione renale rimase stabile, i livelli della proteinuria e della lipidemia si ridussero.

La Tabella I riporta i maggiori reperti istopatologici dei pazienti esaminati. Non c'era correlazione fra le manifestazioni istologiche riportate e la risposta al trattamento. Tutti i pazienti tolleravano bene il trattamento. La perdita di capelli fu severa ma transitoria nei soggetti trattati con CPM giornaliera.

Irreversibile azospermia si manifestò in 6 pazienti. La conta degli spermatozoi cadde da $97.5 \pm 19$ a $1.0 \pm 0.2$ milioni $/ \mathrm{ml}$. Un paziente era azospermico prima del ciclo. Amenorrea (presumibilmente dovuta a fibrosi ovarica) si presentò in quattro pazienti che vennero trattate con terapia ormonale sostitutiva. Un paziente sviluppò iperglicemia, ma non richiese alcun trattamento né riduzione degli steroidi. Il quadro regredì spontaneamente alla fine del trattamento.

Altri effetti collaterali includevano aumento ponderale $(62 \%)$, acne $(17 \%)$, leggero irsutismo $(6 \%)$ e cataratta in due donne. Ipertensione secondaria al trattamento steroideo, si sviluppò in due pazienti e rispose alla sodio-deplezione.

\section{Discussione}

La storia naturale delle GNM non è ancora ben definita. La progressione verso l'insufficienza renale terminale differisce nei diversi lavori pubblicati e i tempi di progressione risultano ugualmente variabili. La remissione spontanea è del $33.9 \%$ a 5.8 anni di follow-up (13), ma anche più elevata per periodi di osservazione in media di 8 e più anni (14).

Donadio et al esaminarono 140 pazienti con GNM: il $36 \%$ sviluppò un'insufficienza renale con una velocità di progressione di 3.6 anni. Tuttavia il $64 \%$ di questi pazienti mantenne una funzione renale stabile. La possibilità di sviluppare insufficienza renale era più elevata in relazione diretta con i livelli di proteinuria e dei trigliceridi, nel sesso maschile, in rapporto alla pressione arteriosa ed a valori inizialmente elevati di creatininemia (15). Per contro altri Autori riportarono una quota favorevole di remissioni spontanee $(16,17)$ indicando un decorso benigno per le GNM non trattate. Tuttavia in accordo con $\mathrm{i}$ criteri da noi usati nel presente studio, il $30 \%$ dei pazienti non trattati ebbe progressione in un pe-

TAB. III - FOLLOW-UP LABORATORISTICO DEI PAZIENTI CON GNM SOTTOPOSTI A TERAPIA IMMUNOSOPPRESSIVA

\begin{tabular}{lccccc}
\hline & \multicolumn{2}{c}{ Iniziale } & \multicolumn{2}{c}{ Fine terapia } & Fine follow-up \\
\hline & \multicolumn{3}{c}{$\mathrm{p}$} & & $\mathrm{p}$ \\
\cline { 2 - 6 } & $114 \pm 82$ & 0.05 & $88 \pm 26$ & $\mathrm{NS}$ & $97 \pm 26$ \\
P creatinina $\mu \mathrm{mol} / \mathrm{L}$ & $4.8 \pm 0.9$ & $<0.01$ & $0.7 \pm 0.3$ & $\mathrm{NS}$ & $0.5 \pm 0.2$ \\
Proteinuria $\mathrm{g} / \mathrm{die}$ & $53 \pm 2$ & $<0.05$ & $66 \pm 3$ & $\mathrm{NS}$ & $68 \pm 2$ \\
Proteinemia $\mathrm{g} / \mathrm{L}$ & $8.88 \pm 3.03$ & $<0.05$ & $6.09 \pm 1.16$ & $\mathrm{NS}$ & $6.33 \pm 2.66$ \\
P colesterolo mmol/L & $2.73 \pm 1.15$ & $<0.05$ & $1.76 \pm 0.45$ & $\mathrm{NS}$ & $1.61 \pm 0.36$ \\
P trigliceridi mmol/L & & & & & \\
\hline
\end{tabular}


riodo di follow-up più breve e solamente il $25 \%$ andò incontro a remissioni spontanee.

I risultati ottenuti da diversi trial terapeutici e le analisi retrospettive dei trattamenti presentano ampia variabilità: nessun beneficio (18), miglioramento nella sopravvivenza (11), completa e parziale remissione (19-20) e riduzione delle progressioni verso l'insufficienza renale terminale (21).

Considerando solo trial prospettici, Kassirer et al ritennero vantaggioso trattare le GNM (22) dal momento che il $71 \%$ dei soggetti non trattati evolvevano verso l'insufficienza renale terminale nell'arco di dieci anni. La letteratura dimostra che col solo prednisone (PDN) non furono ottenuti risultati soddisfacenti (10-1823); tuttavia Hopper et al (24) riportarono un significativo numero di miglioramenti e remissioni ed una più bassa quota di progressioni verso l'insufficienza renale utilizzando alte dosi di steroidi a giorni alterni. Pertanto i risultati negativi di Donadio et al (10) e del gruppo di Toronto (9) potrebbero essere legati allo schema terapeutico utilizzato (basse dosi di PDN e breve periodo di trattamento). Gli agenti alchilanti ugualmente hanno portato a risultati contraddittori. Nei rial controllati il loro effetto era considerato almeno equivalente all'uso del solo steroide (8). Se la malattia è dovuta alla formazione di immunocomplessi solubili, l'associazione fra steroidi ed agenti alchilanti dovrebbe essere molto efficace. Infatti, Ponticelli et al (21) riportarono i migliori risultati nelle GNM con questa associazione in cui, tuttavia, i farmaci venivano usati in sequenza.

Nel presente studio noi abbiamo utilizzato un'effettiva combinazione somministrando PDN e CPM insieme per un periodo medio di 6 mesi. I risultati sono i migliori finora riportati. La quota di remissione era dell' $83 \%$ e nessun paziente andò in dialisi. Il nostro periodo medio di osservazione era di 109 mesi, molto vicino rispetto a quello di Noel et al (14) che riportò una significativa percentuale di progressione verso l'insufficienza renale nell'intero gruppo di pazienti in follow-up. Questo dato non fu osservato in nessuno dei nostri pazienti. I risultati inaspettatamente favorevoli, simili a quelli delle GNMP (25), potrebbero riflettere qualche errore nella selezione dei pazienti che avrebbero potuto presentare una forma non progressiva di GNM. Tut- tavia, tutti i nostri pazienti avevano una sindrome nefrosica con una media di proteinuria di $4.8 \mathrm{~g} /$ die (range 2.5-10 $\mathrm{g} / \mathrm{die}$ ). Solamente tre avevano proteinuria al di sotto di $3 \mathrm{~g} /$ die e le proteine plasmatiche erano 65.55 e $54 \mathrm{~g} / \mathrm{L}$. La classificazione istologica rivelò un ampio spettro di lesioni che includevano modificazioni croniche, essudative e componenti proliferative (Tab. I). Quindi è improbabile che la nostra popolazione comprendesse in prevalenza soggetti con un basso rischio di malattia progressiva. Inoltre vi fu una tendenza alla recidiva che fu riportata solamente in un precedente lavoro (26). Noi abbiamo usato criteri molto rigidi per definire la remissione e la ricaduta, mentre in altre casistiche, nonostante la ricomparsa della proteinuria, situazioni analoghe sarebbero state considerate come miglioramenti rispetto al pre-trattamento. Nel nostro lavoro 9 pazienti recidivarono, corrispondendo al $36 \%$ di tutte le remissioni. Sembrerebbe che l'immunosoppressione nelle GNM possa causare una condizione analoga alla nefrosi lipoidea dei bambini, dove sono frequenti le recidive e la dipendenza steroidea (2). La nostra definizione di ripresa includeva un sedimento anomalo ed una ricomparsa progressiva della proteinuria non necessariamente in range nefrosico. Tuttavia, $\mathrm{SN}$ e/o aumento della creatininemia si presentò in 7 pazienti. Il più interessante risultato fu la stabilizzazione della funzione renale al termine del periodo di follow-up di 109 mesi. Nessun paziente sviluppò insufficienza renale. I pazienti che avevano presentato recidiva furono sottoposti ad un secondo ciclo terapeutico con piena remissione. Gli 8 pazienti non trattati mostrarono, come previsto, un progressivo deterioramento della funzione renale, dal momento che la creatininemia media aumentò da $97 \pm 17$ a $221 \pm 53 \mu \mathrm{mol} / \mathrm{L}$ $(\mathrm{p}<0.05)$ alla fine del follow-up. Cinque progredirono e tre rimasero stabili. La proteinuria rimase essenzialmente immodificata $(3.3 \pm 0.7 \mathrm{Vs} 3.0 \pm 0.5 \mathrm{~g} / \mathrm{die})$. Due dei pazienti che progredirono ed uno che rimase stabile erano $\mathrm{HBsAg}$ positivi. Il trattamento nonostante sia stato ben tollerato, deve comunque essere considerato tossico. Il $40 \%$ dei pazienti sviluppò irreversibile azoospermia o amenorrea. Il regime di PDN a giorni alterni iniziato precocemente limitò gli effetti collaterali degli steroidi rendendo il ciclo meglio tollerato. Noi riteniamo che le alte dosi dei due farmaci o il tempo prolungato di terapia rappresentino il segreto della risposta positiva, dal momento che in passato furono pubblicati risultati meno favorevoli o sfavorevoli con basse dosi (18) o per terapie a breve termine (11) o con singoli farmaci (7-10). Si dovrebbe inoltre enfatizzare che anche $i$ tre pazienti che non andarono completamente in remissione e $\mathrm{i}$ due che progredirono, in accordo con la nostra definizione, mantennero, nonostante tutto, stabile funzione renale ed evitarono l'insufficienza renale terminale. Remissioni intervennero anche in quattro pazienti che avevano IRC prima della terapia, sebbene gli alti valori di creatininemia siano considerati un importante fattore di rischio che limita il trattamento e facilita la progressione della malattia (10-15). Per questa ragione molti Autori non trattano le GNM con incipienti manifestazioni di malattia cronica. Questo è parzialmente supportato dall'evidenza che i processi patofisiologici che portano all'IRC sono differenti dalla malattia da immunocomplessi ( 27 , 28). I nostri dati tuttavia suggeriscono che pazienti con GNM rapidamente evolventi verso la malattia renale cronica possono essere trattati con successo. Questo è in accordo con altri Autori (19) i quali potrebbero non aver avuto i nostri stessi risultati avendo utilizzato dosi ridotte di CPM. I nostri risultati sono infine confermati da quelli ottenuti da $\mathrm{Ki}$ briya et al (20) che riportarono una più bassa percentuale di remissioni con un regime terapeutico simile a più basso dosaggio. In conclusione l'uso del nostro ciclo di terapia immunosoppressiva nel trattamento delle GNM potrebbe essere applicato in quasi tutti i pazienti con glomerulonefriti per evitare la progressione verso l'insufficienza renale terminale. Questo si ottiene nonostante un'elevata quota di recidive che successivamente rispondono con una completa remissione nel $90 \%$ dei casi. Noi non sappiamo se questa incoraggiante risposta possa durare indefinitamente, dal momento che in altri studi le differenze in favore del gruppo trattato erano annullate da un lungo periodo di follow-up (15). Quindi noi dobbiamo essere cauti nell'estendere le nostre conclusioni al di là del nostro periodo di osservazione. Tuttavia in risposta ad un importante quesito presentato recentemente (29), i nostri risultati indicano che tutti i pazienti debbano essere trattati prontamente e ritrattati se re- 
cidivano, dal momento che nelle GNPM le recidive non trattate evolvono rapidamente verso l'uremia (25).

In conclusione è chiaro che questo ed altri trattamenti immunosoppressori sono del tutto sperimentali, e che un trial multicentrico allargato è necessario per stabilirne l'efficacia, il significato dei riscontri istologici apparentemente responsabili della resistenza al trattamento (30) ed il reale bilancio fra la potenziale efficacia ed effetti collaterali (31). Al di fuori di questo studio sperimentale è saggio sospendere gli agenti alchilanti e comunque non utilizzarli in così alto dosaggio, come in questo studio, in pazienti con una proteinuria al di sotto di $3.5 \mathrm{~g} / \mathrm{die}$ e con una creatininemia stabile nel range della norma. Questi pazienti potrebbero essere a basso rischio, avere un incerto beneficio e potrebbero andare incontro a complicanze tardive, ivi comprese alcune malattie neoplastiche.

\section{Riassunto}

Abbiamo studiato l'efficacia di un nuovo protocollo di terapia immunosoppressiva nella Glomerulonefrite Membranosa (GNM). Il trattamento era suddiviso in 4 fasi. 1) Induzione: boli di metilprednisolone associati a dosi orali di ciclofosfamide (CPM). 2) Mantenimento: dosi orali di prednisone (PDN) a giorni alterni associati a dosi gionaliere di CPM. 3) Riduzione: scalo graduale del solo PDN. 4) Sospensione: interruzione della CPM; lenta e graduale riduzione fino alla sospensione del PDN.

Il trattamento durò in media circa 9 mesi (range 6-12). La dose cumulativa del PDN fu di $7.8 \mathrm{~g}$, mentre quella della CPM di 27.5 g. Venticinque dei 30 pazienti trattati ebbero una remissione completa alla fine del ciclo, tre migliorarono e due presentarono una progressione minima con successiva stabilizzazione del quadro clinico. Vi furono dieci recidive di malattia in nove pazienti dopo un periodo medio di 52 mesi dalla fine del ciclo. Tutte le recidive furono ritrattate e 9 pazienti presentarono remissione completa mentre uno migliorò. I risultati ottenuti furono indipendenti dall'istologia renale, dalle concentrazioni della creatinina e dalla proteinuria. In media la creatininemia si ridusse da $114 \pm 82$ $\mu \mathrm{mol} / \mathrm{L}$ a $88 \pm 26 \mu \mathrm{mol} / \mathrm{L}$ al termine $\mathrm{del}$ trattamento e raggiunse $97 \pm 26 \mu \mathrm{mol} / \mathrm{L}$ al termine del follow-up di 109 mesi. L'escrezione urinaria delle proteine fu rispettivamente di $4.8 \pm 0.9,0.7 \pm 0.3 \mathrm{e}$ $0.5 \pm 0.2 \mathrm{~g} / \mathrm{die}$. In un gruppo di pazienti non trattati non vi furono remissioni. La creatininenia aumentò da 97士17 a $221 \pm 53 \mu \mathrm{mol} / \mathrm{L}$ alla fine del periodo medio di follow-up di 5 anni, mentre la proteinuria rimase stabile nel range nefrosico.

Riteniamo pertanto il nostro protocollo terapeutico altamente efficace nell'indurre remissioni nelle GNM e nel prevenire la loro progressione verso l'insufficienza renale terminale.

\section{BIBLIOGRAFIA}

1.

Shalhoub RJ. Pathogenesis of lipoid nephrosis: a disorder of T-cell function. Lancet 1974; 2: 556-9.

2. Siegel NJ, Goldberg B, Krassner LS, Hayslett JP. Long-term follow up of children with steroid responsive nephrotic syndrome. J Pediatr 1972; 8; 251-8.

3. Kincaid-Smith P. The treatment of chronic mesangiocapillary (membranoproliferative) glomerulonephritis with impaired renal function. Med J Aust 1972; 2: 587-92.

4. Steineberg AD. The treatment of lupus nephritis. Kidney Int 1986; 30: 769-87.

5. Newman WJ, Tisher CC, Mc Coy RC, et al. Focal glomerular sclerosis: contrasting clinical paterns in children and adults. Medicine (Baltimore) 1976; 55: 67-87.

6. Cattran DC, Cardella CJ, Roscoe JM, et al. Results of a controlled drug trial in membranoproliferative glomerulonephritis. Kidney Int 1985; 27: 436-41.

7. Ehrenreich T, Porush JG, Churg J, et al. Treatment of idiopathic membranous nephropathy. $\mathrm{N}$. Engl J Med 1976; 295: 741-6

8. Lagrue G, Bernard D, Bariety J, Druett P, Guenel J. Traitement par le Chlorambucil et Azathioprine dans les Glomérulonéphrites primitives: results d'une etude "contrôlée". J Urol Nephrol 1975: 81: $655-72$.

9. Cattran DC, Delmore T, Roscoe J, et al. A randomized controlled trial of prednisone in patients with idiopathic membranous nephropathy. Ann Engl J Med 1989; 320: 210-5.

10. Donadio JV Jr, Torres VE, Velosa JA, et al. Idiopathic membranous nephropathy: the natural history of untreated patients. Kidney Int 1988; 33: 708-15. 
11. Coggins $\mathrm{CH}$, Pinn V, Border $\mathrm{W}$, et al. A controlled study of short-term prednisone treatment in adults with membranous nephropathy. N Engl J Med 1979; 301: 1301-6.

12. Tu WH, Petitti DB, Biava CG, Tulunay Ö, Hopper J Jr. Membranous nephropathy: predictor of terminal renal failure. Nephron 1984; 36: 118-24.

13. Schena FP, Cameron JS. Treatment of proteinuric idiopathic glomerulonephritides in adults: a retrospective survey. Ann J Med 1988; 85: 315-26.

14. Noel LH, Zanetti M, Droz D, Barbanel C. Long-term prognosis of idiopathic membranous glomerulonephritis. Study of 116 untreated patients. Am J Med 1979; 66: 8290 .

15. Pei Y, Cattran D, Greenwood C. Predicting chronic renal insufficiency in idiopathic membranous glomerulonephritis. Kidney Int 1992; 42: 960-6.

16. Davison AM, Cameron JS, Kerr DSN, Oggs SC, Wilkinson RW. The natural History of renal function in untreated idiopathic membranous glomerulonephritis in adults. Clin Nephrol 1984; 22: 61-7.

17. Schiepati A, Mosconi L, Terna A, et al. Prognosis of untreated patients with idiopathic membranous nephropathy. N Engl J Med 1993; 329: 85-9.

18. Black DAK, Rose G, Brewer DB. Controlled trial of prednisone in adults patients with nephrotic Syndrome. Br Med J 1970; 3: 421-6.

19. Alecsopoulos E, Sakellariou G, Mammos D, Karamitsos K, Leontsini M, Papadimitriou M. Cyclophosphamide provides no additional benefit to steroid therapy in the treatment of idiopathic membranous nephropathy. Am J Kidney Dis 1993; 21: 497-503.

20. Kibriya MG, Tishkov I, Nikolov D. Immunosoppressive therapy with cyclophosphamide and predni- solone in severe idiopathic in severe membranous nephropathy. Nephrol Dial Transplant 1994; 9: 138-43.

21. Ponticelli C, Zucchelli P, Passerini $\mathrm{P}$, et al. A randomized trial of methyl prednisolone and chlorambucil in idiopathic membranous nephropathy. N Engl J Med 1989; 320: 8-13.

22. Levey AS, Lau J, Pauker SG, Kassire JP. Idipoathic nephrotic syndrome. Ann Intern Med 1987; 107: 679-713.

23. Pierides AM, Malasit P, Morley AR, Wilkinson R, Uldall PR, Kerr DNS. Idiopathic membranous nephropathy. Q J Med 1977; 46: 163-77.

24. Hopper J, Biava CG, Tu VH. Membranous Nephropathy: high dose alternate-day therapy with prednisone. West J Med 1981; 135 : 1-8.

25. Faedda R, Satta A, Tanda F, Pirisi M, Bartoli E. Immunosoppressive treatment of membranoproliferative glomerulonephritis. Nephron 1994; 67: 59-65.

26. Manos J, Short CD, Acheson EJ, et al. Relapsing idiopathic membranous nephropathy. Clin Nephrol 1982; 18: 286-90.

27. Rossen RD, Reisberg MA, Sharp JT, Suki WN, Schloeder FY, Hill LG. Antiglobulins and glomerulonephritis: classification of the patients by the reactivity of their sera and renal tissue with aggregated and native human IgG. J Clin Invest 1975; 56: 427-33.

28. Kerjaschki D. Molecular pathogenesis of membranous nephropathy. Kidney Int 1992; 41: 1090105.

29. Lewis EJ. Idiopathic membranous nephropathy. To treat or not to treat? N Engl J Med 1993; 329: 127-9.

30. Wakay S, Magil AB. Focal glomerulosclerosis in idiopathic membranous glomerulonephritis. Kidney Int 1992; 41: 428-34.
31. Glassock RJ. Therapy of idiopathic nephrotic syndrome in adults. Am J Nephrol 1993; 13: 422-8. 